\title{
Statistical Description on the Role of Turbulence and Grain Interference on Particle Entrainment from Gravel Beds
}

DOI:

10.1061/(ASCE)HY.1943-7900.0001224

\section{Document Version}

Accepted author manuscript

Link to publication record in Manchester Research Explorer

\section{Citation for published version (APA):}

Cecchetto, M., Tregnaghi , M., Bottacin Busolin, A., Tait, S., \& Marion, A. (2016). Statistical Description on the Role of Turbulence and Grain Interference on Particle Entrainment from Gravel Beds. Journal of Hydraulic Engineering, [06016021]. https://doi.org/10.1061/(ASCE)HY.1943-7900.0001224

\section{Published in:}

Journal of Hydraulic Engineering

\section{Citing this paper}

Please note that where the full-text provided on Manchester Research Explorer is the Author Accepted Manuscript or Proof version this may differ from the final Published version. If citing, it is advised that you check and use the publisher's definitive version.

\section{General rights}

Copyright and moral rights for the publications made accessible in the Research Explorer are retained by the authors and/or other copyright owners and it is a condition of accessing publications that users recognise and abide by the legal requirements associated with these rights.

\section{Takedown policy}

If you believe that this document breaches copyright please refer to the University of Manchester's Takedown Procedures [http://man.ac.uk/04Y6Bo] or contact uml.scholarlycommunications@manchester.ac.uk providing relevant details, so we can investigate your claim.

\section{OPEN ACCESS}




\title{
STATISTICAL DESCRIPTION ON THE ROLE OF TURBULENCE AND GRAIN INTERFERENCE ON PARTICLE ENTRAINMENT FROM GRAVEL BEDS
}

\author{
Martina Cecchetto ${ }^{1}$, Matteo Tregnaghi ${ }^{2}$, Andrea Bottacin-Busolin ${ }^{3}$, Simon Tait ${ }^{4}$ and Andrea Marion ${ }^{5}$
}

\section{Abstract}

A complete understanding of the role of grain-scale particle-flow interaction in sediment entrainment and transport has still not been achieved in spite of recent technological advancement in measurement capabilities. In this study the initial motion of natural sediment particles in a gravel deposit was detected and combined with simultaneous local measurements of the velocities on a horizontal plane located above the bed surface using a three-component stereoscopic PIV. A series of experimental tests with increasing low values of boundary shear stress were conducted. The acquisition system allowed coupling between streamwise and vertical near-bed velocity and the entrainment of more than 900 individual grains. Initial analysis agreed with previous observations on the predominance of sweeps (Quadrant IV) and to a lesser extent of outward interactions (Quadrant I) in entraining gravel particles. However, the latter were found to move sediments just as efficiently as sweeps impacting on particles that had long periods of rest and so were exhibiting higher levels of stability. This behavior suggests that sweep-induced lift based on Bernoulli's principle does not entirely explain the generation of vertical forces on highly stable bed particles. Closer inspection of the data revealed that many entrainments were correlated to occasions when stable bed grains interacted with grains travelling in their close vicinity. Around $30 \%$ of the entrained population was observed to initiate motion in this type of situation. For this subsample of entrainment events outward interactions were found to be comparatively more effective than for the non-interference case, while the relative contribution of sweeps exhibited an opposite trend.

Keywords: Quadrant Analysis, Entrainment, Turbulence.

\footnotetext{
${ }^{1} \mathrm{PhD}$ student, Dept. of Civil and Structural Engineering, University of Sheffield, Sheffield, UK.

${ }^{2}$ Senior scientist, Dept. of Industrial Engineering, University of Padua, Padua, Italy.

${ }^{3}$ Lecturer, School of Mechanical, Aerospace and Civil Engineering, University of Manchester, Manchester, UK.

${ }^{4}$ Professor, Dept. of Civil and Structural Engineering, University of Sheffield, UK

${ }^{5}$ Associate Professor, Dept. of Industrial Engineering, University of Padua, Italy
} 


\section{INTRODUCTION}

It is broadly recognized that grain entrainment occurs when pressure gradients around a surface particle generate drag and lift forces of sufficient magnitude and duration that exceed some critical threshold thus dislodging grains from their rest position. The turbulent nature of the local flow field, although organized in repeating and coherent flow patterns (Kline et al. 1967), and the evidence that the forces that resist motion are not a simple function of the submerged grain weight (Kirchner et al. 1990) are two key elements that render the entrainment of sediments in rivers an intermittent and almost random process as documented by Drake et al. (1988). The pioneering studies by Einstein (1950), Sutherland (1967) and Grass (1970) have contributed to the development of modeling approaches that described particle entrainment using probabilistic concepts (e.g., Cheng and Chiew 1998; Papanicolaou et al. 2002; Wu and Chou 2003; Hofland and Battjes 2005), and technical capabilities achieved in the last two decades have now created the possibility to test such concepts. Recently, two additional aspects were experimentally investigated that characterize physical interactions at the sediment-flow interface. First, it was clarified that the duration of the hydrodynamic forces, not only their magnitude, is important in determining whether a grain will be entrained, thus arguing that a threshold criterion would be better described by an impulse rather than a force balance (Diplas et al. 2008; Celik et al. 2010; Valyrakis et al. 2010). Second, while most past investigations have assumed turbulent flow and bed particle arrangement to be statistically independent, evidence was found that they have dependence (Papanicolaou et al. 2001; Dey et al. 2011a; Tregnaghi et al. 2012a).

There still exist questions on the relative contribution that different types of coherent flow structures, defined through the decomposition of the Reynolds Stress into four quadrants, hereafter denoted as Q-I, Q-II, Q-III and Q-IV (Lu and Willmarth 1973), actually play in terms of particle entrainment. While Sutherland (1967) formerly recognized the importance of flow structures on the initial motion of sediments, Heathershaw and Thorne (1985) and Nelson et al. (1995) were among the first who experimentally demonstrated a quantitative correspondence between turbulent bursts 
and the dislodgment of particles from the bed surface. Both studies reported that the pickup of sediments was found to be correlated to the instantaneous value of the streamwise velocity (Q-I and Q-IV events), although sweeps (Q-IV) were considered to be predominant due to their higher frequency of occurrence. More recently, Dwivedi et al. (2011a) established that sweeps were associated with higher-magnitude lift and drag forces than those associated with outward interactions (Q-I), thus suggesting the latter were of less importance in supplying bedload flux. Similar conclusions can be found in Dey et al. (2011b), however earlier observations by Heathershaw and Thorne (1985) revealed that outward interactions, although weaker and less frequent than sweeps, are still capable of causing sediment movement at intermediate (i.e., not extreme) values of the local velocity fluctuations.

These findings partly reflect a lack of a complete understanding of the processes that generate hydrodynamic forces on grains. While it is quite well accepted that the instantaneous drag force strongly correlates well with the instantaneous streamwise velocity (Schmeeckle et al. 2007), the mechanism contributing to the lift term lacks such a complete understanding. Detert et al. (2010) found that high-speed fluid patterns were associated with pressure-drop events, which is consistent with the suggestion that sweeps are the dominant cause of bedload flux. On the other hand, Schmeeckle et al. (2007) reported that the vertical force was not dominantly determined by Bernoulli pressure differences across the particle as they measured poor correlation between the streamwise velocity and lift force, as observed also by Dwivedi et al. (2011b) for low particle exposures. This uncertainty is dramatically increased by the evidence that the rate of grain entrainment is highly sensitive to very modest changes in flow conditions for near-threshold conditions (Nelson et al. 1995; Sumer et al. 2003).

In all these studies velocity or force measurements were made at a single point or on a solitary particle having fixed or unnatural pocket geometry. No spatial observations were made of the fluid velocities linked to the initial grain motion over natural beds, and little attention was given to the role of local surface irregularities in the response of different grains to turbulent events generating 
similar-magnitude drag and lift forces. This concept was first illustrated by Grass (1970) explaining that the entrainment of particles is more likely to occur as the overlap increases between the probability distributions of the hydrodynamic forces and of the resisting forces opposing to motion. Evidence was provided by Papanicolaou et al. (2001), who obtained single-point velocity measurements directly correlated with the movement of a 'target' particle displaced over three different regularly-packed surfaces. They demonstrated that these different arrangements affected significantly the frequency and magnitude of turbulent events and, in turn, the probability of sediment entrainment. Further observations by Dey et al. (2011b, 2012) confirmed that near-bed turbulence parameters exhibited dissimilar patterns caused by differences in bed conditions.

The experiments described in the present note were focused on providing a more complete explanation of the range of individual grain entrainments observed from deposits of natural gravel and on the specific influence of the local flow field. The experiments were designed so that grain entrainments could be clearly identified and linked to simultaneous measurements of the near-bed local flow field. The statistical analysis of grain motion data and fluctuations of the local flow field was coupled with information on the relative stability of bed particles, estimated based on the recorded duration of their resting periods and on direct observations of the interference generated by particles travelling in the close vicinity of grains that were entrained.

\section{EXPERIMENTAL EQUIPMENT AND PROCEDURE}

A 12-m-long and 0.46-m-wide tilting laboratory flume was used for the experiments. The initial 1.5$\mathrm{m}$-long reach contained a static gravel surface, the remaining flume length was filled with river gravel with mean size $d_{50}=5.0 \mathrm{~mm}$ and standard deviation $\sigma_{g}=1.3 \mathrm{~mm}$, creating a well-mixed deposit with no appreciable bed forms. No sediment was artificially fed into the flume and the bed was gradually water-worked by the flow thus removing unnatural grain pocket geometries. All the measurements were collected within 30 minutes from the start of the experiment before degradation occurred and the surface particle arrangement significantly changed. The same procedure ensured that similar water-working conditions were established in all tests, which were run with constant relative 
submergence by setting the water depth to $h_{u}=100 \mathrm{~mm}$. Tests with increasing boundary shear stress levels were achieved by adjusting the slope and flow rate to attain uniform flow. Conditions ranging from close to the threshold of motion to up to 1.5 times the critical Shields' parameter $\tau^{*}{ }^{*}=0.057$ (Shields 1936) were used. Table 1 shows the main hydraulic parameters for the three tests analyzed, namely $\mathrm{T} 1, \mathrm{~T} 2$ and $\mathrm{T} 3$.

A stereoscopic two-camera PIV (Particle Image Velocimetry) system was used allowing for the reconstruction of the three-component velocity vector on a horizontal plane above the bed. This was combined with a synchronized third camera devoted to grain tracking. The recording system measured above a bed area of $220 \mathrm{~mm}$ by $40 \mathrm{~mm}$ located on the centerline of the flume $6.70 \mathrm{~m}$ from the inlet as illustrated in Figure 1a. The size of the observation area was selected as a compromise between attaining a sediment-size spatial image resolution and detecting a statistically significant number of grains over a representative area of the bed surface. A glass sheet was located at the selected water depth to prevent image distortion due to water surface oscillations. The floating panel was designed such as to produce negligible interference as it was typically immersed by $<2$ $\mathrm{mm}$ and caused no larger surface waves in the wake than those generated by the free water surface, without significantly affecting the flow in the near bed as discussed by Detert et al. (2010). Although variations in turbulence intensity cannot be excluded, data presented in next sections reveal typical features of unaltered flows over rough boundaries.

The two PIV cameras-2 and -3 recorded image pairs in double-frame mode (time delay between frames $\left.\Delta t_{L}=2 \mathrm{~ms}\right)$ of neutrally buoyant seeding particles $\left(d_{p}=200 \mu \mathrm{m}, \rho_{p}=995 \mathrm{~kg} / \mathrm{m}^{3}\right)$ at a frequency $f_{R}=45 \mathrm{~Hz}$. Two pulsed lasers (Nd:YAG) with expanding optics generated a high-intensity light sheet approximately $\Delta Z_{L}=3-\mathrm{mm}$-thick located parallel and at $Z_{L}=8 \mathrm{~mm}$ above the original sediment bed. The vertical position of the field of view was selected such as to avoid disturbances with the bed roughness elements and with the bedload flux which was observed to form roughly one-diameterthick layer, but well within the wall-shear layer. The thickness of the light sheet was adjusted so as to obey the $1 / 4$ rule (Adrian 1991), i.e. the maximum vertical displacement of seeding particles was less 
than about $0.25 \cdot \Delta Z_{L}$ to minimize out-of-plane loss of particles. Each image pair was divided into interrogation areas that corresponded to a physical area of approximately $4.3 \times 4.3 \mathrm{~mm}$, with a $75 \%$ overlap resulting in a square mesh grid comprised of $N_{G}=35 \times 185=6475$ nodes, corresponding to a velocity measurement spatial resolution of $1.1 \mathrm{~mm}$ in both the streamwise and lateral directions. Figure $1 \mathrm{~b}$ depicts the location of the velocity measurements over the sediment background in order to illustrate the spatial location of the velocity vectors compared to the position of the bed particles. Due to geometrical constraints, the opening angle between the two PIV cameras was approximately 32 degrees, this resulted in the measurement precision of the out-of-plane component to be less accurate compared to typical error estimates reported by, e.g., Dwivedi et al. (2011) for twocomponent PIV. Details of the reconstruction geometry and relevant error analysis are reported in the Appendix.

The bed grain camera-1 was synchronized with the PIV recording system and focused on the sediment bed surface in order to detect individual grain motion. The bed was illuminated by a white strobe light that was triggered at the leading edge of the second laser pulse such as the image of the bed was captured at the time of the second PIV frame. Individual grain entrainment, movement and deposition were identified by direct inspection of the image sequences. The grain diameter $d$ and the position of the grain centroid $\left(X_{G}, Y_{G}\right)$ along its motion was determined through the identification of a bounding box around the grain at each time step. Initial inspection of the images revealed that a significant proportion of grains (approx 30\%) were observed to be entrained due to collision with or interference by other moving grains, while some grains were observed to leave their rest position a few frames (around a few hundredths of a second) after the passage of a moving grain in their vicinity (i.e., within 1-diameter distance).

In total 18000 frames for the three experiments (corresponding to approximately 2 minutes for each test) were processed resulting in nearly 900 identified entrainment events. Closer inspection of bed images revealed that some grains were considered at rest while they were actually re-arranging their positions by shaking and rolling around their center, thus not fully achieving a proper condition 
of rest. A threshold equal to $0.2 \mathrm{~s}$ (corresponding to 10 frames) was imposed as the minimum rest time to exclude these 'biased' entrainment events from the statistics. The next section discusses how grain displacements were associated with the near-bed flow field by correlating individual entrainment events to the 'instantaneous' velocity vector fluctuations measured at the closest position of the projection of the grain centroid on the laser plane, i.e. at $\left(X_{G}, Y_{G}, Z_{L}\right)$.

\section{ANALYSIS OF NEAR-BED FLOW AND GRAIN ENTRAINMENT}

\section{Double-averaged Turbulence Intensity and Conditional Reynolds Shear Stresses}

The PIV dataset includes the time series of the streamwise, lateral and stream-normal velocity components (namely $u, v$ and $w$ ) that are also distributed over the whole area of observation. Each component $x$ can be decomposed as $x=\langle\bar{x}\rangle+\tilde{x}^{\prime}$, where $\langle\bar{x}\rangle$ is the double-averaged mean and $\tilde{x}^{\prime}$ is the fluctuating term over finite time and spatial scales. A summary of the double-averaged mean and of the root mean square (RMS, or standard deviation for zero mean) of the fluctuating term, $\tilde{x}_{R M S}^{\prime}$, is presented in Table 2 for each velocity component. Given the relatively long velocity time series with imposed uniform flow depth, the size of the area of observation, and the statistically homogenous irregularities of the bed surface (i.e., uniformly-distributed sediment with no bed forms developed during the experiments), it can be reasonably assumed that over the $X$ and $Y$ coordinates $\langle\bar{x}\rangle \cong \bar{x}(X, Y)$ and $\tilde{x}^{\prime} \cong x^{\prime}(X, Y)$ where $\bar{x}$ is the time-averaged mean and $x^{\prime}$ the time-fluctuating term at a given position. This assumption is found to hold as the RMS of the difference $\left(\tilde{x}^{\prime}-x^{\prime}\right)$ is less than about $8 \%$ of the RMS of $\tilde{x}^{\prime}$ for all the three velocity components and for all tests. For comparison, values of $\tilde{u}_{R M S}^{\prime}$ are slightly less than 1.7-1.8 the shear velocity, which is consistent with vertical profiles of time-averaged turbulence intensity proposed by Kironoto and Graf (1994) and following approximations by Nikora and Goring (2000) at normalized distance from the mean bed approximately $Z_{L} / h_{U} \approx 0.10$. The ratio $\tilde{v}_{R M S}^{\prime} / \tilde{u}_{R M S}^{\prime}$ that characterizes flow anisotropy is close to 0.70 as proposed by Nezu and Nakagawa (1993), while $\tilde{w}_{R M S}^{\prime} / \tilde{u}_{R M S}^{\prime}$ slightly overestimates the theoretical value of 0.55 , which is possibly due to the larger error associated with the vertical component. The 
where $\lambda_{j}=1$ if $\left|u^{\prime} w^{\prime}\right|>H\left(\tilde{u}_{R M S}^{\prime} \tilde{w}_{R M S}^{\prime}\right)$ and $\lambda_{j}=0$ otherwise; $N=$ number of measurements; $H=$ holeanalysis, the product of the RMS of the time-spatial fluctuations is selected as a scale parameter. 

at a maximum distance of $0.7 \mathrm{~mm}$ from the grain's centroid projection on the laser plane for the entire duration of its motion. The time series of the velocity data were extracted from the PIV flow field records up to the instant in which the grain was entrained by the flow. This was determined by examining the records of images from the grain camera-1, and then tracking the particle motion back to the time of first detected particle movement. The most representative velocity values for the entrainment events were selected based on previous observations by Nelson et al. (1995) who found higher correlation with velocities measured approximately $0.1 \mathrm{~s}$ before transport occurred. Denoting with $t_{\text {start }}$ the time when the grain was observed to initiate its motion, the velocity measurements were extracted at $t_{\text {start }}-2 \Delta t, t_{\text {start }}-\Delta t$, and $t_{\text {start }}$, where $\Delta t=1 / f_{R}=0.022 \mathrm{~s}$. In the present experiments the duration of a bursting event was approximately $t_{E}=0.05 \mathrm{~s}$ (compares well with, e.g., Nikora and Goring 2000; Dey et al. 2011b), i.e. $t_{E} \approx 2 \Delta t=0.044 \mathrm{~s}$. Figure $2 \mathrm{~b}$ illustrates the distribution of entrainment events associated with the $u^{\prime}$ and $w^{\prime}$ pairs computed as the average value over the period $2 \Delta t$ preceding the event. This is superimposed to the curves delimiting the hyperbolic hole regions which are defined for consistency with the present approach as:

for the hole size parameter $H$ ranging from 0 to 8 . In order to enhance the accuracy of the match between the type of flow structure and the relevant particle motion, all entrainment events were excluded that were associated with flow velocities falling within the range $\left|w^{\prime}\right|< \pm \varepsilon_{w}$ and $\left|u^{\prime}\right|< \pm \varepsilon_{u}$ where $\varepsilon_{w}$ and $\varepsilon_{u}$ are the random error estimates of the vertical and streamwise components respectively (see Appendix). The diagram shows a predominance of sweeps and, to a lesser extent, of outward interactions in generating sediment motion. The frequency of occurrence of the four types of coherent structures associated with an entrainment event is reported in Table 3. The proportion 


$$
S_{i, H}=\frac{1}{\left\langle\overline{u_{i}^{\prime} w_{i}^{\prime}}\right\rangle N} \sum_{j}\left(u_{i}^{\prime} w_{i}^{\prime}\right) \lambda_{j}
$$
where (Nikora and Goring 2000):

$50 \%$ of flow events associated with entrainment is caused by sweeps with outward interactions causing more bedload entrainments than the combination of all ejections and inward interactions.

Figure $3 \mathrm{~b}$ compares the conditional relative magnitude $\left|S_{i, H}\right|$ of each type $i$ of structure within the fluid with the conditional relative magnitude of the structures associated with an entrainment event,

with $\left\langle\overline{u^{\prime} w^{\prime}}\right\rangle$ being the scaling shear stress term averaged over the area of observation (Table 2), and $N$ the number of measurements or of detected particle entrainments. Figure 3a supports previous reporting that for weakly mobile beds within the wall-shear layer $\left(Z_{L} / h_{U} \approx 0.10\right)$ the opposing events are approximately balanced, with both interactions becoming less significant for $H>4$, while sweeps and ejections vanishing for $H>8$ (Dey et al. 2012). Approaching closer the top of the bed particles $\left(Z / h_{U} \approx 0.02\right.$, data not available in this study) would reveal increasing relative magnitude of $Q$-IV events, with the contributions from Q-I and Q-III events becoming more feeble. However, Figure 3b shows an alternate conclusion in that the higher the value of $\left|S_{i, H}\right|$ the more likely for a particle to be entrained, as in this latter case outward interactions become relatively more important, although sweeps still predominate. Denoting now $E_{i}$ as the fraction of sediment entrained by the $i$-type structure, we define the ratio $\eta_{i}=E_{i} / T_{i}$ as the entrainment efficiency. Comparison between this and previous studies reported in Table 3 provides evidence that although sweeps move more sediment than outward interactions, individually, the latter exhibit comparable or even higher efficiencies.

\section{Conditional Turbulence Intensity and Grain Stability and Interference Effects}

High streamwise velocity fluctuations have been previously recognized to play a major role in grain entrainment. However results reported in this and in previous research are conflicting with other more recent works that see sweeps as the dominant mechanism as they are not only more frequent but also because are capable to generate larger form drag and lift due to Bernoulli pressure 
difference on a particle (e.g., Detert et al. 2010; Dwivedi et al. 2011a; Dwivedi et al. 2011b). Here the conditional turbulence intensity of the flow structures leading to an entrainment event is correlated with additional information on the relative stability of and on the interference effects on the bed particles that were entrained during the period of observation.

Visual examination of records of images from camera-1 and direct observations during the experiments revealed that grain motion became apparent in isolated spots or in the form of intermittent clusters of few particles, while general mobilization of the so called active layer was never attained. The detected mobile population was found to have periods of rest, $t_{R E S T}$, before reentrainment following a decaying exponential distribution with mean rest time $\mu_{R E S T} \approx 3.5-4.0 \mathrm{~s}$, with less than $10 \%$ of the sample resting on the bed for times longer than approximately $10-12 \mathrm{~s}$. As Wu and Yang (2004) observed that the mean resting time was longer for the more sheltered particles to the flow, following a similar argument led us to identify those particles with longer resting periods as having a higher ability to oppose the destabilizing action exerted by the flow. Closer image inspection revealed that a significant number of grains, including approximately $30 \%$ of the entrained population, initiated their motion immediately after the passage of other particles travelling in their close vicinity. Such an entrainment occurrence could be caused by grain collision, however the frequency of the acquisition system did not allow the actual moment of impact to be observed. This type of event was defined as a grain-to-grain interference.

Figure 4a displays the variation of the conditional turbulence intensity $u_{R M S}^{\prime}$ with the hole-size parameter $H$ as a function of the resting period $\left(t_{\text {REST }} \geq t^{*}\right.$, with $t^{*}$ threshold selected based on the probability distribution of the sample) of the entrained particles for coherent structures of type I (outward interactions) and of type IV (sweeps) associated with an entrainment event without grain interference (the no interference case). An interesting feature is that $U_{R M S, I V}^{\prime}$ increases up to $20-25 \%$ for longer rest times $\left(t_{R E S T} \geq 8 \mathrm{~s}\right.$ ) compared to the whole examined population (corresponding to $t_{R E S T}$ $\geq 0.2 \mathrm{~s}$ ), suggesting that sweeps dislodge the more stable particles by producing larger form drag and Bernoulli's lift. On the other hand $u_{R M S, I}^{\prime}$ does not exhibit any significant signature with varying the 
rest time, i.e. with varying the forces opposing to the motion of bed particles, resulting that the normalized difference of the streamwise turbulence intensity between Q-IV and Q-I, defined as $\Delta u_{R M S}^{\prime}=\left(u_{R M S, I V}^{\prime}-u_{R M S, I}^{\prime}\right) / \tilde{u}_{R M S}^{\prime}$, increases up to $50 \%$ for $t_{R E S T} \geq 8 \mathrm{~s}$ (see Figure $4 \mathrm{~b}$ ). Sweeps were found to maintain fairly constant or slightly decreasing efficiency $\left(\eta_{I V}=1.50\right.$ to 1.60$)$ in removing the more sheltered particles by strengthening those forces that are commonly associated with higher streamwise velocity fluctuations. This is consistent with the mechanism described by Dwivedi et al. (2011a). Unexpectedly, the entrainment efficiency of outward interactions was found to increase for longer particle rest times, with $\eta_{\text {, }}$ ranging approximately from 1.35 up to 1.70 for $t_{R E S T} \geq 8 \mathrm{~s}$ (see Table 4). The result has no obvious explanation, thus the same analysis was performed for the conditional turbulence intensity due to the vertical component. Figure 5a depicts a reverse behavior compared with the former case as $w_{R M S, I V}^{\prime}$ tends to decrease for longer resting periods, which indicates a suppression of the wake term contributing negatively to the total lift and counteracting the effect of Bernoulli's lift as discussed by Dwivedi et al. (2011b). Conversely $w_{R M S, I}^{\prime}$ is seen to slightly increase with the time of rest, causing the difference of the relative vertical turbulence intensity between Q-IV and Q-I, namely $\Delta w_{R M S}^{\prime}=\left(w_{R M S, I V}^{\prime}-w_{R M S, I}^{\prime}\right) / \tilde{w}_{R M S}^{\prime}$, to slowly reduce by up to $-40 \%$ for $t_{R E S T} \geq 8 \mathrm{~s}$ (Figure 5b). Whether this evidence can provide sufficient justification for the observed increase of entrainment efficiency for outward interactions is not readily apparent and it will be discussed in more detail in the next section.

A similar analysis was carried out only for those grains that were seen to initiate motion after interference caused by other moving particles. Table 4 shows the entrainment efficiency for coherent structures of type I (outward interactions) and of type IV (sweeps) as a function of $t_{\text {REST }}$. Compared with the no interference case no correlation was observed between $w_{R M S, I V}^{\prime}$ and $w_{R M S, I}^{\prime}$, respectively, and the resting periods of the particles (data not presented). The only observed significant signature was an increase of $w_{R M S, I V}^{\prime}$ and $w_{R M S, I}^{\prime}$ with respect to the relevant 'no interference' events, with both intensities ranging from $0.15 \mathrm{~m} / \mathrm{s}$ for $H=0$ to $0.25 \mathrm{~m} / \mathrm{s}$ for $H=4$. The entrainment efficiencies of both 
types of structures remain relatively constant up to $t_{R E S T} \geq 4$, for longer rest times outward interactions become more and more effective while sweeps become less efficient. The analysis further validates the predominance of sweeps, which combined together with outward interactions account for more than $75 \%$ of the entrainment events. However, outward interactions are seen to contribute globally more effectively compared with the no interference case, with relative proportion of entrained sediment $E_{l}$ increasing from $26.9 \%$ to $31.3 \%$; conversely sweeps are less effective with relative proportion of entrained sediment $E_{/ V}$ reducing from $47.1 \%$ to $43.7 \%$.

\section{DISCUSSION}

The evidence that particles having longer resting periods are associated with increasing $u_{R M S, I V}^{\prime}$ combined with reduced $W_{R M S, I V}^{\prime}$ is consistent with the mechanism suggested in recent studies that sweeps induce dislodgment of particles by causing larger combined form drag and Bernoulli's lift compared with outward interactions. This does not provide sufficient justification for regarding sweeps as the only dominant structure inducing the entrainment of bed particles. Following on Grass' concept of overlapping probabilities their relative stability also plays a major role (e.g., Bottacin-Busolin et al. 2008; Tregnaghi et al. 2012b). A large fraction of less stable particles are moved by forces that have sufficiently larger magnitude than those generated by the mean flow but relatively smaller than the relevant extreme values, which, in turn, lead both sweeps and outward interactions to attain comparable entrainment efficiencies. A more subtle result is the observed increase of the efficiency of outward interactions with increasing particle stability. A possible explanation is that the vertical force is not adequately predicted solely by Bernoulli pressure differences.

According to Schmeeckle et al. (2007), an approximation of the hydrodynamic force $\mathbf{F}_{H}$ exerted on the particle by the fluid can be obtained from the momentum balance of a particle:

$\mathbf{F}_{H}=\frac{1}{2} \rho C_{D} A|\mathbf{U}| \mathbf{U}+\rho_{s} V C_{L} \cdot \mathbf{U} \times \mathbf{\Omega}+\rho_{s} V\left\langle\frac{D \mathbf{U}}{D t}\right\rangle_{V}$ 
$\left\langle\frac{D w}{D t}\right\rangle_{v}=\left\langle\frac{\partial w}{\partial t}+u \frac{\partial w}{\partial X}+v \frac{\partial w}{\partial \gamma}+w \frac{\partial w}{\partial Z}\right\rangle_{v}$

$F_{H, Z}=\frac{1}{2} \rho C_{D} A \bar{u} \cdot w+\rho_{S} V C_{L} \frac{\partial \bar{u}}{\partial z} \cdot u$ responsible for such departure. found in previous studies. Noting that

where $A$ and $V$ are the projection area and the volume of the particle, respectively; $\mathbf{U}$ and $\boldsymbol{\Omega}$ the total velocity and vorticity of the fluid; $C_{D}$ and $C_{L}$ empirical drag and lift coefficients; $D / D t$ and $\langle\cdot\rangle_{V}$ denote, respectively, material derivative and average over the particle volume. The three terms on the righthand side are the hydrodynamic drag, the hydrodynamic lift, and a force that arises from the acceleration of the fluid. Further assumptions on dominant terms lead the vertical components of the first two forces of Eq.(4) to be approximated as (van Radecke and Schulz-DuBois 1988):

Here the first term on the right-hand side represents the quasi-steady lift due to vertical velocity fluctuations (namely the wake-induced lift) and the second term is Bernoulli's lift. Dwivedi et al. (2011b) demonstrated that the latter has significantly higher magnitude compared with the wakeinduced lift, however both Schmeeckle et al. (2007) and Dwivedi et al. (2011b) claimed the concurrence of both positive and negative lift fluctuations at times of increased streamwise velocity, which contrasts with the simple model given by Eq.(5). They argued that either the acceleration force or the form lift produced by particle shape, which is not accounted for in Eq.(4), were supposedly

If a turbulent structure produces high pressure on the bottom of the particle and a low pressure on the top, the vertical acceleration force is large and a high-lift event will occur, although any discussion on this term is rather speculative at this stage as no clear experimental evidence can be

one may argue that the coherence of the flow structure limits the effects of the local acceleration $\partial w / \partial t$ over the time scale of the turbulent event, and that the highest convective acceleration of $w$ is expected towards the vertical direction. Although mathematically non-negative at the bed, for $Z>0$ 
IV) fluxes, while it gives a negative contribution for upward decelerating or downward accelerating

352 movements of the fluid mass. This would provide at least qualitative justification for the evidence that opposing trends of $w_{R M S, I V}^{\prime}$ and $w_{R M S, I}^{\prime}$, respectively, with the variation of the particle rest time are associated with opposing trends of the relevant entrainment efficiencies. This is further supported by the observation that interfering particles cause an increased turbulence intensity of the vertical fluctuations, which may be responsible of the counteracting effects due to the acceleration force for sweeps and outward interactions, respectively, resulting in an increasing efficiency gap. In their experiments Nelson et al. (1995) created strong gradients in the flow by introducing a backward step upstream the measurement point. In presence of the step, they found that outward interactions increased their effectiveness in moving grains. Here grain interference has similar effects to those due to the upstream flow separation induced by the backward step, which for the most interfering case led to entrainment efficiencies $\eta_{I}=2.45$ and $\eta_{I V}=1.45$ (Run 1 in Nelson et al. 1995). Similar high (Q-I) and low (Q-IV) values were found also in the experiments by Heathershaw and Thorne (1985), here reported in Table 3 for comparison, where interference effects were possibly amplified by the presence of poorly sorted sediment, with the largest particles facilitating the dislodgment of the more sheltered fine particles causing local vertical accelerations of the fluid mass.

\section{CONCLUSIONS}

Experiments were carried out linking the near-bed flow field and individual entrainment events from a gravel deposit under weak sediment transport conditions. Results confirmed earlier observations on the predominance of sweeps (Q-IV) and to a lesser extent of outward interactions (Q-I) in dislodging gravel particles, although approximately $30 \%$ of the observed entrainment events were caused by a grain-to-grain interaction. Clear signatures in the turbulence intensity validated previous findings on the correlation between sweeps and entrainment events based on the current understanding of the forces that are most likely to generate particle motion. However, the reported discrepancies with the hypothesis that sweeps are the prevailing mechanism are explained through 
376 the evidence of concurrent factors: (1) similar to the Grass' concept of overlapping probabilities, a

377 significant fraction of less stable particles are moved by smaller hydrodynamic forces than their 378 relevant extreme values; (2) there is no clear consensus that Bernoulli's lift can entirely explain the 379 formation of vertical forces, while evidence is given in this study that force that arises from the 380 acceleration of the local mass of fluid may also play a role; (3) moving particles can induce 381 interference effects by producing an increased turbulence intensity of the vertical fluctuations 382 potentially associated with the vertical fluid acceleration and the occurrence of high lift events able 383 to trigger sediment motion. Such a grain interference phenomenon has counteracting effects on 384 sweeps and outward interactions, respectively, resulting in the latter to comparatively increase their 385 relative efficiency, which would explain previous experimental observations leading to similar results 386 as those reported in this study. 


\section{Appendix}

The reconstruction geometry of the image acquisition system is here represented by a pinhole camera model having angle of view $2 \vartheta_{X}=10$ degrees and sensor size $\left(D_{X}, D_{Y}\right)=1600 \times 300$ pixels, resulting in:

$$
D=\frac{D_{X} / 2}{\tan \vartheta_{x}}
$$

and

$$
2 \vartheta_{Y}=\arctan \frac{D_{Y} / 2}{D}
$$

where $D$ is the sensor distance from the lens and $2 \vartheta_{y}$ is the angle of view in the $Y$-direction (Fig. 6 ). Denoting with $d=(d x, d z)$ the distance of the lens from the field of view, the size of the area of observation along the streamwise and crosswise directions is given as:

$$
L_{x}=2\left[d x-d z \tan \left(\alpha-\vartheta_{x}\right)\right]
$$

and

$$
L_{y}=2 d \tan \vartheta_{y}
$$

The two PIV cameras were mounted at a distance $d=1456 \mathrm{~mm}$ from the bed with an opening angle $2 \alpha=32$ degrees, such as that the area of observation of each camera was $259-\mathrm{mm}$-long and $48-\mathrm{mm}$ wide. This extension was then reduced to the intersection of the sampling areas of the two camera views corresponding approximately to $220 \times 40 \mathrm{~mm}$. The minimum detectable displacement along the $X$-direction, $\Delta x_{\min }$, resulted in the maximum uncertainty associated with the streamwise component given as:

$\varepsilon_{u}= \pm \frac{\Delta x_{\min }}{\Delta t_{L}}= \pm \frac{d z}{\Delta t_{L}} \tan \left(\alpha+\arctan \frac{\Delta x_{p}}{D}\right) \mp \frac{d x}{\Delta t_{L}}$

where $\Delta x_{p}=0.15$ pixels is the estimated sub-pixel accuracy of the cross-correlation algorithm (Raffel et al. 2007), and $\Delta t_{L}=2 \mathrm{~ms}$ the time between laser pulses. The error estimates for the three velocity component are then $\varepsilon_{u}= \pm 12.4 \mathrm{~mm} / \mathrm{s}, \varepsilon_{v}= \pm 11.9 \mathrm{~mm} / \mathrm{s}$ and $\varepsilon_{w}= \pm 43.5 \mathrm{~mm} / \mathrm{s}$ respectively, where: 
$410 \quad \varepsilon_{v}= \pm \frac{\Delta y_{\min }}{\Delta t_{L}}= \pm \frac{d \cdot \Delta x_{p} / D}{\Delta t_{L}}$

411 and

$412 \varepsilon_{w}= \pm \frac{\Delta z_{\min }}{\Delta t_{L}}= \pm \frac{d z}{\Delta t_{L}} \mp \frac{d x}{\Delta t_{L}} \tan \left(\frac{\pi}{2}-\alpha-\arctan \frac{\Delta x_{p}}{D}\right)$

\section{Acknowledgments}

414 The research leading to these results has received funding from the People Programme (Marie Curie

415 Actions) of the European Union's Seventh Framework Programme under the Initial Training Network

416 (FP7-PEOPLE-2012-ITN) HYTECH 'Hydrodynamic Transport in Ecologically Critical Heterogeneous

417 Interfaces', N.316546. 


\section{References}

Adrian, R. J. (1991), Particle-imaging techniques for experimental fluid mechanics, Ann. Rev. Fluid Mech., 23, 261-304

Bottacin-Busolin, A., S. J. Tait, A. Marion, A. Chegini, and M. Tregnaghi (2008), Probabilistic description of grain resistance from simultaneous flow field and grain motion measurements, Water Resour. Res., 44, W09419, doi:10.1029/2007WR006224.

Celik, A. O., P. Diplas, C. L. Dancey, and M. Valyrakis (2010), Impulse and particle dislodgement under turbulent flow conditions, Phys. Fluids, 22(4), 046601.

Cheng, N. S., and Y. N. Chiew (1998), Pickup probability for sediment entrainment, J. Hydraul. Eng., 124(2), 232-235.

Detert, M., V. Weitbrecht, and G. H. Jirka (2010), Laboratory measurements on turbulent pressure fluctuations in and above gravel beds, J. Hydraul. Eng., 136(10), 779-789.

Dey S., R. Das, R. Gaudio, S. K. Bose (2012), Turbulence in mobile-bed streams, Acta Geophysica, 60(6), 15471588.

Dey, S., S. Sarkar, S. Bose, S. Tait, and O. Castro-Orgaz, (2011a), Wall-wake flows downstream of a sphere placed on a plane rough wall, J. Hydraul. Eng., 137(10), 1173-1189.

Dey, S., S. Sarkar., and L. Solari (2011b), Near-bed turbulence characteristics at the entrainment threshold of sediment beds, J. Hydraul. Eng.,137(9), 945-958.

Diplas, P., C. L. Dancey, A. O. Celik, M. Valyrakis, K. Greer, and T. Akar, (2008), The role of impulse on the initiation of particle movement under turbulent flow conditions, Science, 322, 717-720.

Dwivedi, A., B. Melville, A.Y. Shamseldin, and T. K. Guha (2011a), Flow structures and hydrodynamic force during sediment entrainment, Water Resour. Res., 47,W01509.

Dwivedi, A., B. Melville, A. Y. Shamseldin, and T. K. Guha (2011b), Analysis of hydrodynamic lift on a bed sediment particle, J. Geophys. Res., 116, F02015.

Drake, T. G., R. L. Shreve, W. E. Dietrich, P. J. Whiting, and L. B. Leopold (1988), Bedload transport of fine gravel observed by motion-picture photography, J. Fluid Mech., 192, 193-217.

Einstein, H. A. (1950). The bed-load function for sediment transportation in open channel flows. Tech. Bull. 1026, 78 pp., Soil Conserv. Serv., U.S. Dep. of Agric., Washington, D. C. 
Grass, A. J. (1970), Initial instability of fine sand, J. Hydraul. Div., 96(3), 619-632.

Heathershaw, A. D., and P. D. Thorne (1985), Sea-bed noises reveal role of turbulent bursting phenomenon in sediment transport by tidal currents, Nature, 316, 339-342, doi:10.1038/316339a0.

Hofland B., and A. J. Battjes (2005), Probability density function of instantaneous drag forces and shear stresses on a bed J. Hydraul. Eng., 132(11), 1169-1175.

Kirchner, J. W., W. E. Dietricht, F. Iseya, and H. Ikeda (1990), The variability of critical shear stress, friction angle, and grain protrusion in water-worked sediments, Sedimentology, 37, 647-672.

Kironoto, B. A., and W. H. Graf (1994), Turbulence characteristics in rough uniform open-channel flow, Proc. Ice Water Mar. Energy, 106, 333-344.

Kline, S. J., W. C. Reynolds, F. A. Schraub, and P. W. Runstadler (1967), The structure of turbulent boundary layers, J. Fluid Mech., 30(4), 741-773.

Lu S. S., and W. W. Willmarth (1973), Measurement of the structure of the Reynolds stress in a turbulent boundary layer, J. Fluid Mech., 60, 481-511

Nelson, J. M., R. L. Shreve, S. R. Mclean, and T. G. Drake (1995), Role of near-bed turbulence structure in bed load transport and bed form mechanics, Water Resour. Res., 31(8), 2071-2086, doi:10.1029/95WR00976.

Nezu, I., and H. Nakagawa (1993), Turbulence in Open-Channel Flows, Balkema, Rotterdam, Netherlands.

Nikora, V., and D. Goring (2000), Flow turbulence over fixed and weakly mobile gravel beds, J. of Hydraul. Eng., 126(9), 675-690.

Papanicolaou, A., P. Diplas, C. Dancey, and M. Balakrishnan (2001), Surface roughness effects in near-bed turbulence: Implications to sediment transport, J. Eng. Mech., 127(3), 211-218, doi:10.1061/(ASCE)0733-9399(2001)127:3(211).

Papanicolaou, A., P. Diplas, N. Evaggelopoulos, and S. Fotopolous (2002), Stochastic incipient motion criterion for spheres under various bed packing conditions, J. of Hydraul. Eng., 128(4), 369-380, doi:10.1061/(ASCE)0733-9429(2002)128:4(369).

Raffel, M., C. E. Willert, S. T. Wereley, and J. Kompenhans (2007), Particle Image Velocimetry: A Practical Guide, Springer, New York. 
Shields, A. (1936), Application of similarity principles and turbulence research to bed-load movement, Hydrodynamics Laboratory Publ. No. 167, W. P. Ott, and J. C. van Uchelen, trans., U.S. Dept. of Agr., Soil Conservation Service Cooperative Laboratory, California Institute of Technology, Pasadena, Calif.

Schmeeckle, M. W., J. M. Nelson, and R. L. Shreve (2007), Forces on stationary particles in near-bed turbulent flows, J. Geophys. Res., 112, F02003, doi:10.1029/2006JF000536.

Sumer, B.M., L. H. C. Chua, N. S. Cheng, and J. Fredsoe (2003), Influence of turbulence on bed load sediment transport, J. Hydraul. Eng., 129(8), 585-596.

Sutherland, A. J. (1967), Proposed mechanism for sediment entrainment by turbulent flows, J. Geophys. Res., 72(24), 6183-6194.

Tregnaghi, M., A. Bottacin-Busolin, A. Marion, and S. Tait (2012a), Stochastic determination of entrainment risk in uniformly sized sediment beds at low transport stages: 1. Theory, J. Geophys. Res., 117, F04004, doi: 10.1029/2011JF002134.

Tregnaghi, M., A. Bottacin-Busolin, S. Tait, and A. Marion (2012b), Stochastic determination of entrainment risk in uniformly sized sediment beds at low transport stages: 2. Experiments, J. Geophys. Res., 117, F04005, doi: 10.1029/2011JF002134.

Valyrakis, M., P. Diplas, C. L. Dancey, K. Greer, and A. O. Celik (2010), The role of instantaneous force magnitude and duration on particle entrainment, J. Geophys. Res., 115, F02006.

van Radecke, H., and E. O. Schulz-DuBois (1988), Linear response of fluctuating forces to turbulent velocity components, in Applications of Laser Anemometry to Fluid Mechanics, edited by R. J. Adrian et al., pp. 23-44, Springer, Berlin.

Wu, F. C., and Y. J. Chou (2003), Rolling and lifting probabilities for sediment entrainment, J. Hydraul. Eng., 129(2), 110-119.

Wu, F. C., and K. H. Yang (2004), A stochastic partial transport model for mixed-size sediment. Application to assessment of fractional mobility, Water Resour. Res., 40, W04501. 
Table 1. Hydraulic Parameters for the three tests. $S=$ slope of the flume; $Q=$ steady flow discharge; $U=$ depth averaged flow velocity; $R_{E}=R_{H} U / v=$ flow Reynolds number, with $R_{H}=$ hydraulic radius; $u_{s}=\left(g R_{H} S\right)^{0.5}=s h e a r$ velocity, with $g=$ acceleration due to gravity; $\tau^{*}{ }_{0}=\tau_{0} / g\left(\rho_{s}-\rho\right) d_{50}=$ Shields parameter, where $\tau_{0}=$ boundary shear stress and $\rho_{S}$ and $\rho=$ density of sediment and water respectively; $R_{E, D}=d u_{s} / v=$ grain Reynolds number; $Q_{S}=$ sediment discharge.

\begin{tabular}{ccccccccc}
\hline Test & $\begin{array}{c}S \\
{[\%]}\end{array}$ & $\begin{array}{c}Q \\
{[\mathrm{l} / \mathrm{s}]}\end{array}$ & $\begin{array}{c}U \\
{[\mathrm{~m} / \mathrm{s}]}\end{array}$ & $\begin{array}{c}R_{E}\left(10^{5}\right) \\
{[-]}\end{array}$ & $\begin{array}{c}u_{s} \\
{[\mathrm{~m} / \mathrm{s}]}\end{array}$ & $\begin{array}{c}\mathrm{\tau}^{*} \\
{[-]}\end{array}$ & $\begin{array}{c}R_{E, D} \\
{[-]}\end{array}$ & $\begin{array}{c}Q_{S} \\
{[\mathrm{~g} / \mathrm{s}]}\end{array}$ \\
\hline $\mathrm{T} 1$ & 0.65 & 44.8 & 0.97 & 0.78 & 0.074 & 0.068 & 370 & 3.1 \\
$\mathrm{~T} 2$ & 0.77 & 48.1 & 1.05 & 0.84 & 0.080 & 0.080 & 400 & 3.4 \\
T3 & 0.83 & 49.8 & 1.08 & 0.86 & 0.083 & 0.086 & 420 & 4.0 \\
\hline
\end{tabular}


Table 2. Summary of double-averaged mean and of the root mean square (RMS) of the fluctuating terms.

\begin{tabular}{ccccccccccc}
\hline \multirow{2}{*}{ Test } & $\begin{array}{c}\langle\bar{u}\rangle \\
{[\mathrm{m} / \mathrm{s}]}\end{array}$ & $\begin{array}{c}\langle\bar{v}\rangle \\
{[\mathrm{m} / \mathrm{s}]}\end{array}$ & $\begin{array}{c}\langle\mathrm{w}\rangle \\
{[\mathrm{m} / \mathrm{s}]}\end{array}$ & $\begin{array}{c}\tilde{u}_{R M S}^{\prime} \\
{[\mathrm{m} / \mathrm{s}]}\end{array}$ & $\begin{array}{c}\tilde{v}_{R M S}^{\prime} \\
{[\mathrm{m} / \mathrm{s}]}\end{array}$ & $\begin{array}{c}\tilde{w}_{R M S}^{\prime} \\
{[\mathrm{m} / \mathrm{s}]}\end{array}$ & $\begin{array}{c}\left(u^{\prime}-\tilde{u}^{\prime}\right)_{R M S} \\
{[\mathrm{~m} / \mathrm{s}]}\end{array}$ & $\begin{array}{c}\left(v^{\prime}-\tilde{v}^{\prime}\right)_{R M S} \\
{[\mathrm{~m} / \mathrm{s}]}\end{array}$ & $\begin{array}{c}\left(w^{\prime}-\tilde{w}^{\prime}\right)_{R M S} \\
{[\mathrm{~m} / \mathrm{s}]}\end{array}$ & $\begin{array}{c}-\left\langle\overline{u^{\prime} w^{\prime}}\right\rangle \\
{\left[\mathrm{m}^{2} / \mathrm{s}^{2}\right]}\end{array}$ \\
\hline T1 & 0.70 & 0.003 & 0.000 & 0.130 & 0.080 & 0.104 & 0.011 & 0.004 & 0.008 & 0.0041 \\
T2 & 0.74 & -0.002 & -0.001 & 0.124 & 0.078 & 0.095 & 0.009 & 0.002 & 0.007 & 0.0044 \\
T3 & 0.75 & -0.013 & -0.005 & 0.132 & 0.079 & 0.100 & 0.005 & 0.002 & 0.004 & 0.0051 \\
\hline
\end{tabular}


Table 3. Relative duration $T_{i}$ (average from all tests) and relevant occurrences of entrainment $E_{i}$ associated with individual flow structures for the three tests and average from all tests $\left(\eta_{i}=E_{i} / T_{i}\right)$.

\begin{tabular}{cccccccccccc}
\hline $\begin{array}{c}\text { Type of } \\
\text { structure }\end{array}$ & \multicolumn{3}{c}{ Flow events $T_{i}[\%]$} & \multicolumn{3}{c}{ Entrainment events $E_{i}[\%]$} & \multicolumn{3}{c}{ Efficiency $\eta_{i}[-]$} \\
\hline Q-I & 1 (1) & (2) & (3) & (4) & T1 & T2 & T3 & All & $(1)$ & $(2)$ & $(3)$ \\
Q-II & 29.8 & 20.8 & 16.3 & 21.5 & 28.7 & 26.0 & 27.8 & 28.0 & 1.42 & 1.98 & 1.28 \\
Q-III & 19.3 & 21.4 & 17.3 & 15.4 & 8.8 & 10.4 & 8.4 & 9.0 & 0.45 & 0.52 & 0.57 \\
Q-IV & 31.0 & 28.7 & 31.3 & 29.7 & 44.3 & 54.2 & 45.8 & 46.4 & 1.50 & 1.24 & 1.37 \\
\hline
\end{tabular}

(1) This study; (2) Heathershaw and Thorne (1985); (3) Nelson et al. (1995); (4) Papanicolaou et al. (2001) 
Table 4. Efficiency versus rest time for coherent structures of type I (Outward Interactions) and of type IV (Sweeps).

\begin{tabular}{ccccccc}
\hline Rest time & \multicolumn{2}{c}{ No grain interference } & \multicolumn{3}{c}{ Grain interference } \\
$t_{R E S T} \geq t^{*}[\mathrm{~s}]$ & $\eta_{\mathrm{IV}}[-]$ & $\eta_{\mathrm{I}}[-]$ & $\eta_{\mathrm{IV}}[-]$ & $\eta_{\mathrm{I}}[-]$ & $W_{R M S, I V}^{\prime}[\mathrm{m} / \mathrm{s}]$ & $W_{R M S, I}^{\prime}[\mathrm{m} / \mathrm{s}]$ \\
\hline$t^{*}=0.2 \mathrm{~s}$ & $1.52(47.3 \%)$ & $1.36(26.9 \%)$ & $1.41(43.7 \%)$ & $1.58(31.3 \%)$ & $0.159-0.248$ & $0.143-0.225$ \\
$t^{*}=1.0 \mathrm{~s}$ & 1.62 & 1.38 & 1.40 & 1.84 & $0.156-0.248$ & $0.143-0.216$ \\
$t^{*}=2.0 \mathrm{~s}$ & 1.53 & 1.51 & 1.45 & 1.81 & $0.144-0.225$ & $0.150-0.216$ \\
$t^{*}=4.0 \mathrm{~s}$ & 1.51 & 1.52 & 1.39 & 1.80 & $0.141-0.237$ & $0.153-0.222$ \\
$t^{*}=6.0 \mathrm{~s}$ & 1.45 & 1.61 & 1.26 & 1.91 & $0.138-0.237$ & $0.155-0.236$ \\
$t^{*}=8.0 \mathrm{~s}$ & 1.47 & 1.69 & 1.21 & 1.99 & $0.138-0.249$ & $0.152-0.234$ \\
\hline
\end{tabular}




\section{List of Figures}

Figure 1. (a) Bed sketch of the observed area. (b) PIV mesh grid with nodes location.

Figure 2. (a) Joint frequency distribution of $u^{\prime}$ and $w^{\prime}$ for run T1. Percentages of time occupied by each type of structure are: Q-I $=20.2 \%, \mathrm{Q}-\mathrm{II}=29.1 \%, \mathrm{Q}-\mathrm{III}=20.4 \%, \mathrm{Q}-\mathrm{IV}=$ 30.3\%. (b) Entrainment events caused by flow events with no interference (black dots) and with grain interference (grey dots).

Figure 3. (a) Variation of the relative magnitude $\left|S_{i, H}\right|$ with hole-size parameter $H$ for each type $i$ of structure within the fluid. (b) Variation of the relative magnitude $\left|S_{i, H}\right|$ with hole-size parameter $H$ for each type $i$ of structure associated with entrainment events.

Figure 4. (a) Variation of turbulence intensity $u_{R M S}^{\prime}$ with the hole-size parameter $H$ as a function of the rest period $\left(t_{R E S T} \geq t^{*}\right)$ for coherent structures of type I (outward interactions) and of type IV (sweeps) associated with an entrainment event (no interference). (b) Normalized difference of turbulence intensity measured at entrainment events for outward interactions and sweeps as a function of the rest period of the entrained particles $\left(t_{\text {REST }} \geq t^{*}\right)$.

Figure 5. (a) Variation of turbulence intensity $w_{R M S}^{\prime}$ with the hole-size parameter $H$ as a function of the rest period $\left(t_{\text {REST }} \geq t^{*}\right)$ for coherent structures of type I (outward interactions) and of type IV (sweeps) associated with an entrainment event (no interference). (b) Normalized difference of turbulence intensity measured at entrainment events for outward interactions and sweeps as a function of the rest period of the entrained particles $\left(t_{R E S T} \geq t^{*}\right)$.

Figure 6. Basic stereoscopic imaging configuration using angular lens displacement. 


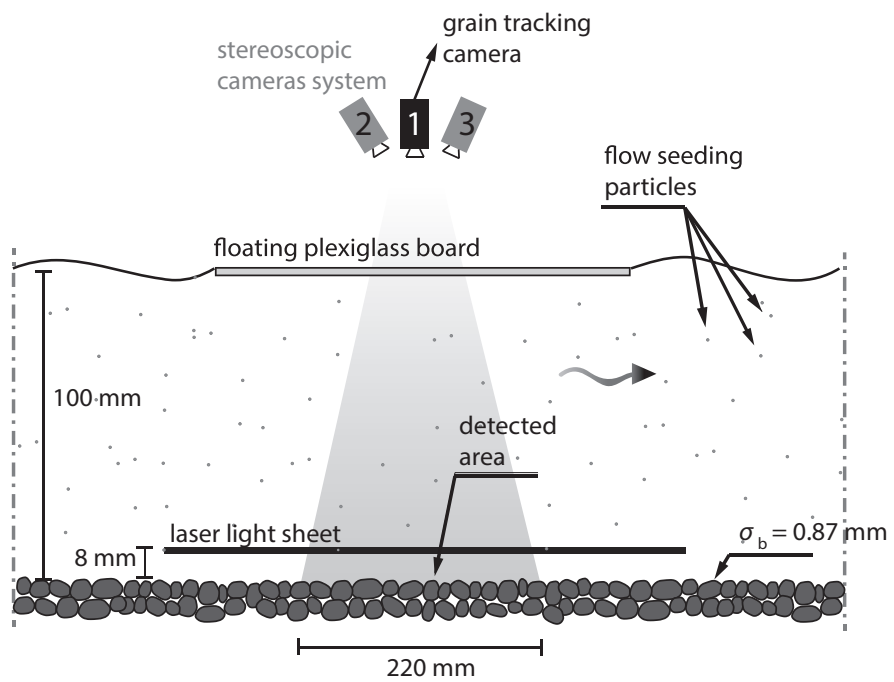

(a)

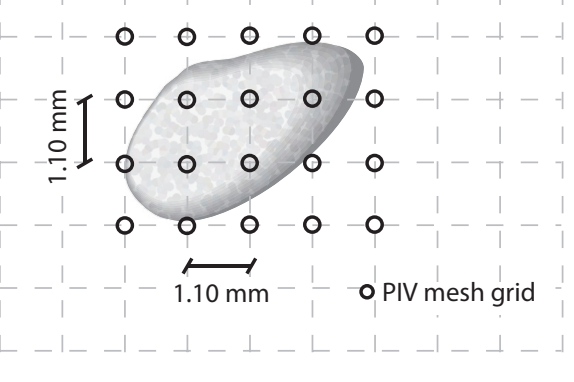

(b)

Figure 1 
(a)

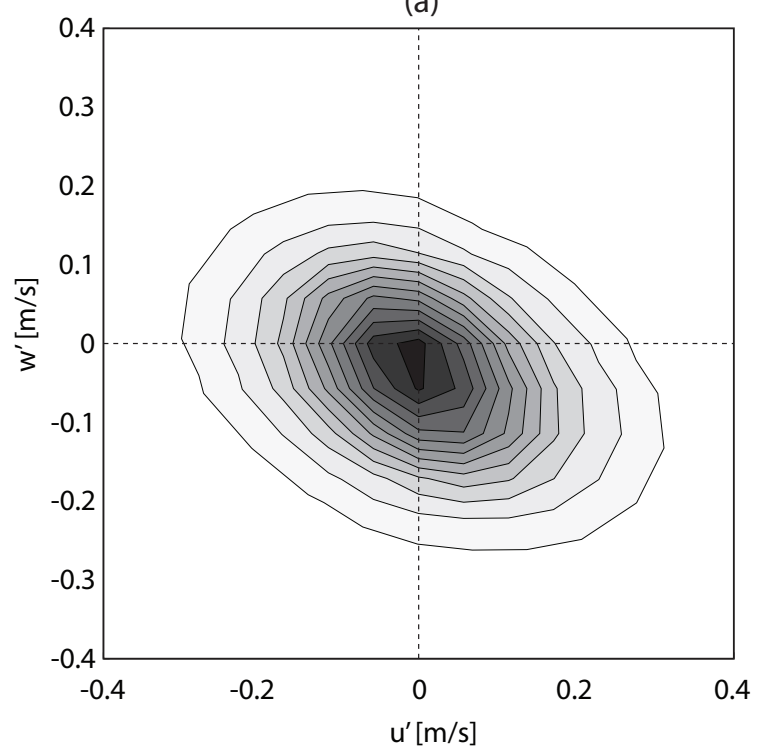

(b)

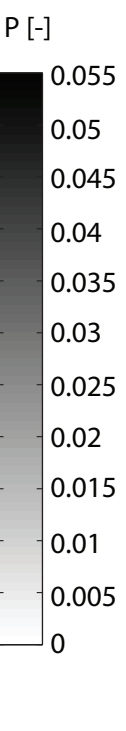

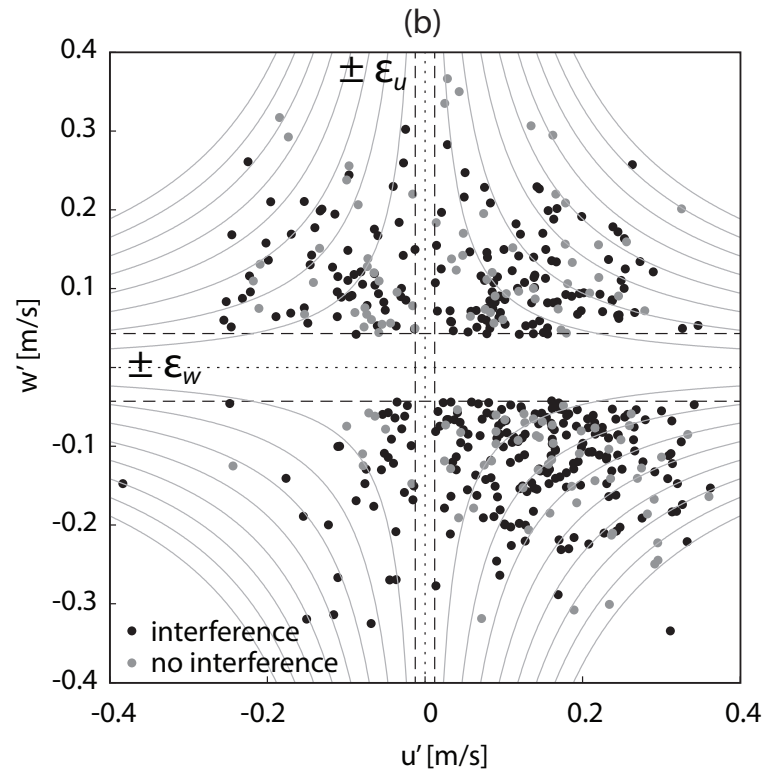

Figure 2 
(a) Flow

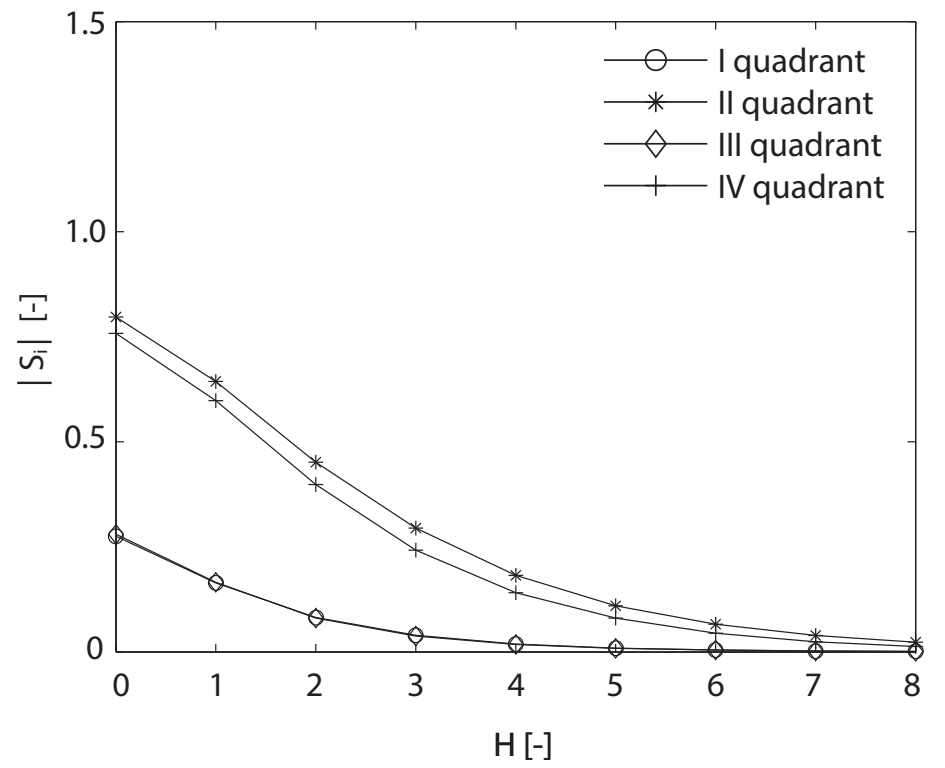

(b) All entrainments

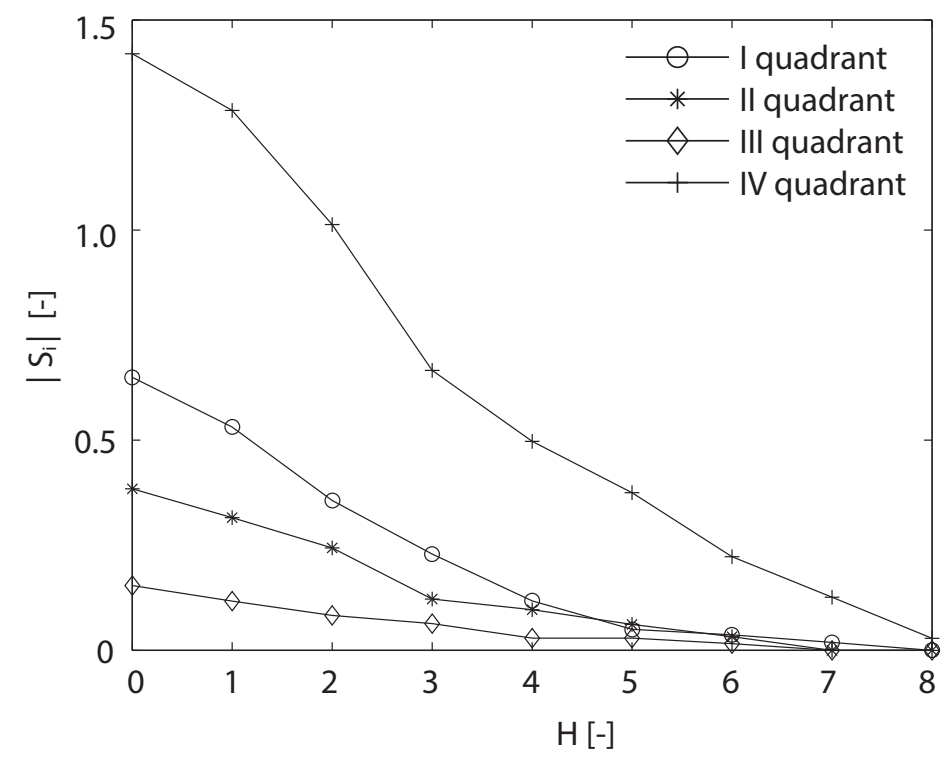

Figure 3 
(a)

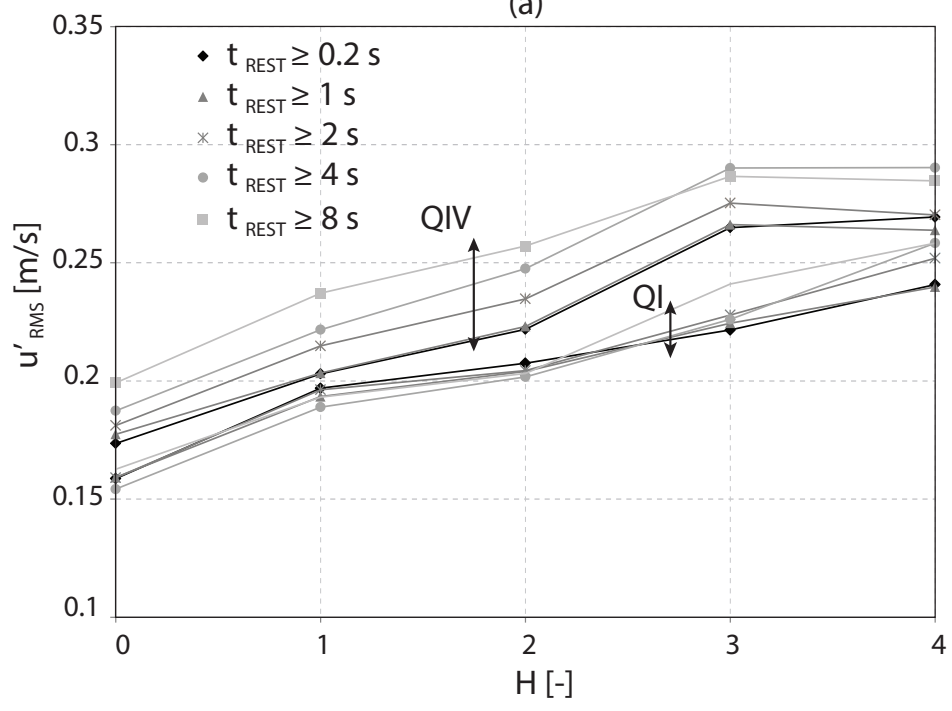

(b)

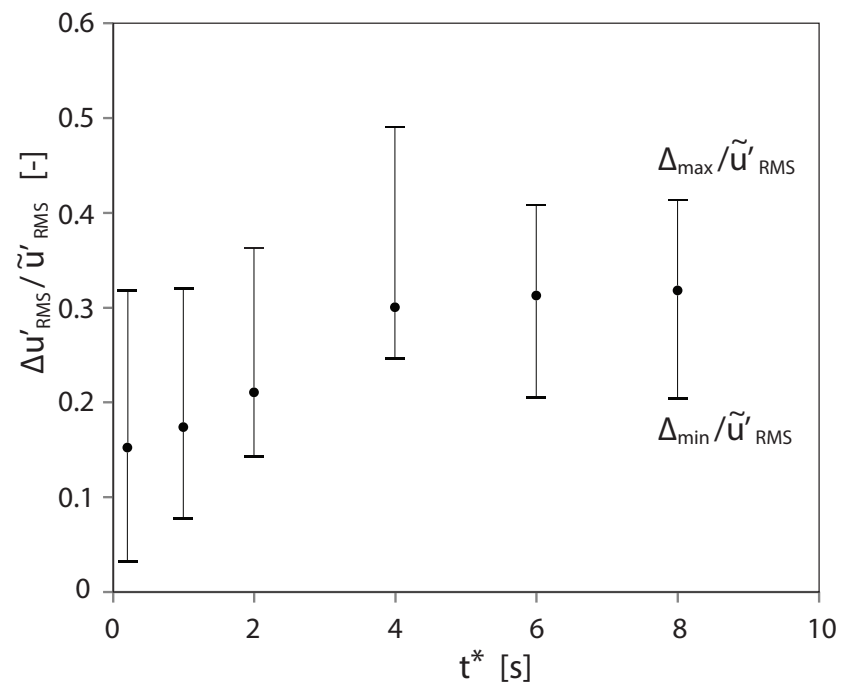

Figure 4 
(a)

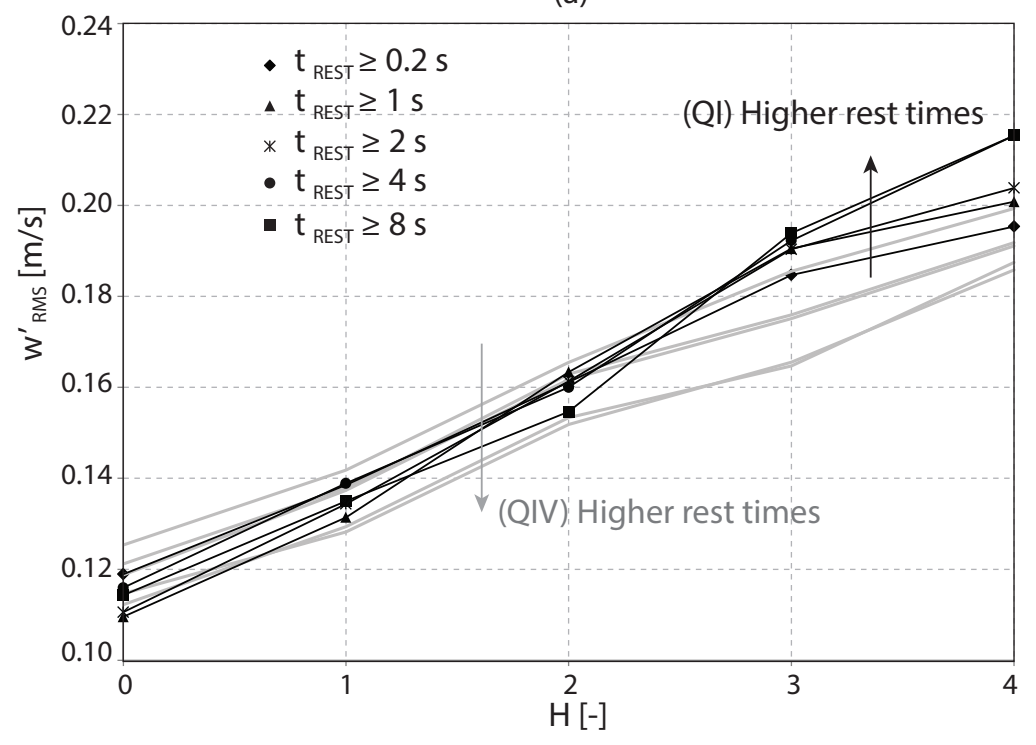

(b)

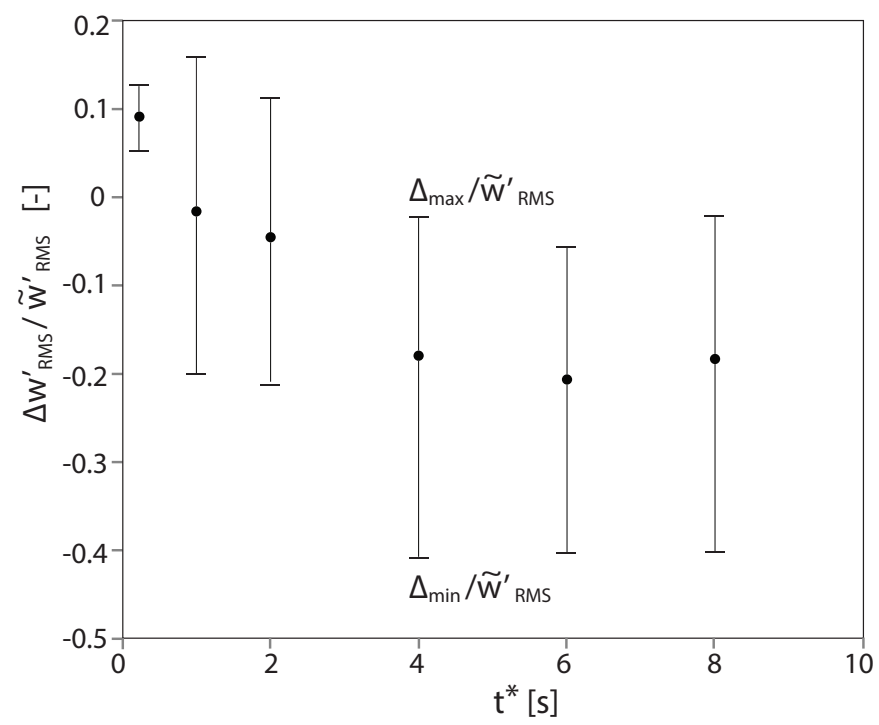

Figure 5 


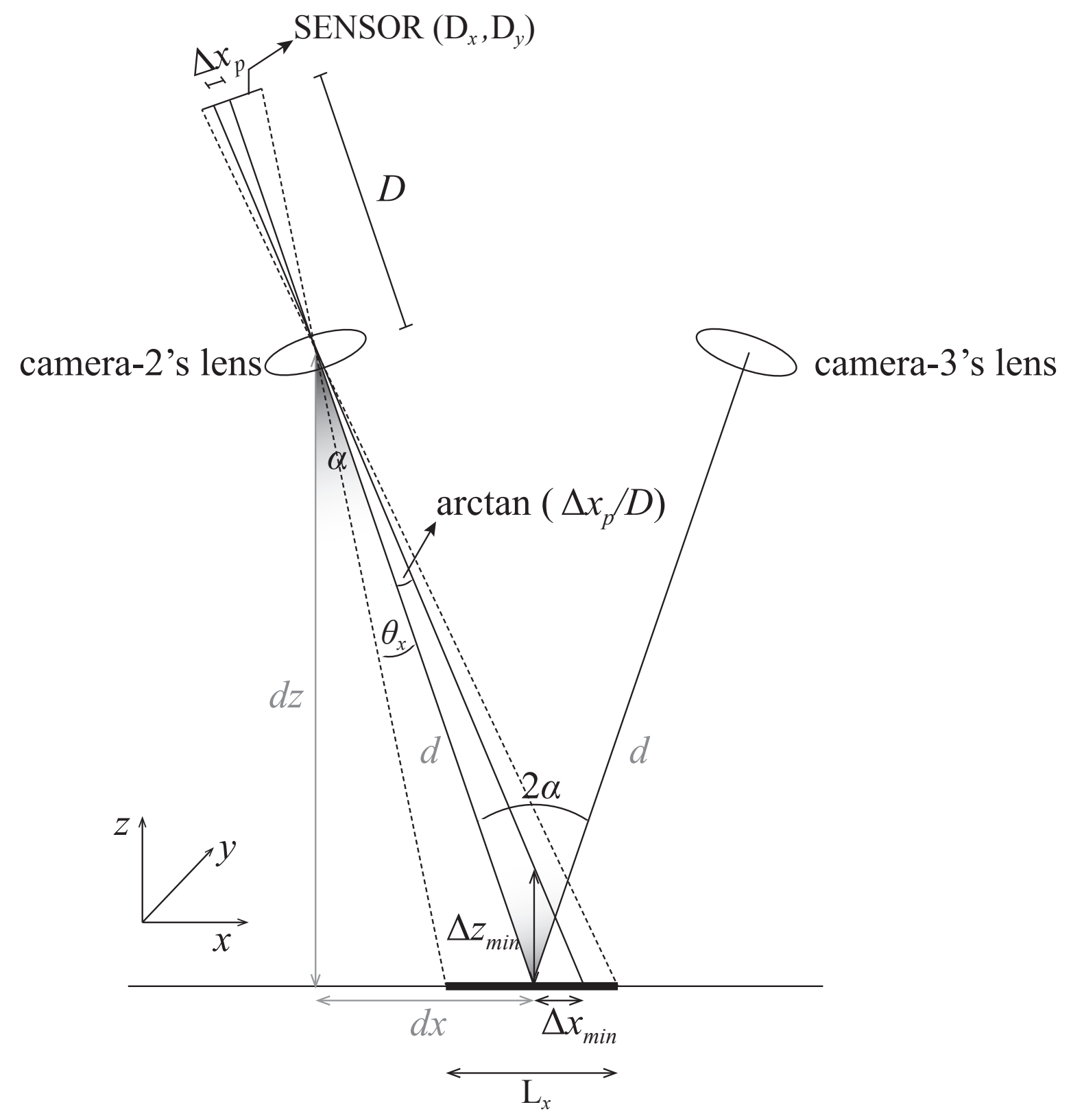

Figure 6 